\title{
An empirical scaling for deuterium retention in co-deposited beryllium layers
}

\author{
G. De Temmerman ${ }^{1}$, M.J. Baldwin ${ }^{1}$, R.P. Doerner ${ }^{1}$, D. \\ Nishijima $^{1}$, and K. Schmid ${ }^{2}$ \\ ${ }^{1}$ Center for Energy Research, University of California at San Diego, La Jolla, \\ CA-92093, USA \\ ${ }^{2}$ Max-Planck Institut für Plasmaphysik, EURATOM Association, D-85748, Garching \\ b. München, Germany \\ E-mail: gdetemmerman@ucsd.edu
}

\begin{abstract}
.
Different mechanisms will contribute to tritium retention in ITER, amongst which co-deposition with materials from the plasma-facing components may be the main contributor. A systematic study of the influence of the deposition conditions (substrate temperature, deposition rate, energy of the incident particles) on the deuterium retention in co-deposited beryllium layers has been carried out in PISCES-B. The mechanism by which deuterium co-deposits with beryllium appears to be a combination of co-deposition and implantation, with a decreased retention for increased deposition rate and an increased retention for increased incident deuterium particles energy. A scaling equation is developed, providing a method to predict the retention in Be codeposits formed in PISCES-B as a function of the layer formation conditions. Using this equation, previously published data on retention in Be co-deposits are re-examined and relatively good agreement is found with the prediction of the scaling equation.
\end{abstract}

PACS numbers: 52.40.Hf, 52.77.Dq 


\section{Introduction}

The minimization of fuel retention, in next step fusion devices, will be one of the more challenging problems to the realization of burning plasma reactors. In ITER, such in-vessel retention is to be limited to only $350 \mathrm{~g}$ of tritium [1]. Retention is not an operational issue in present day devices for several reasons. Firstly, the main working gas is mostly pure deuterium (only JET and TFTR used mixtures of deuterium and tritium) as a fuel, and secondly, tritium fuel, where injected is limited to only 0.01-0.2 $\mathrm{g}$ per pulse (to be compared to $50 \mathrm{~g}$ of tritium to be injected per ITER pulse)[2].

In ITER, different mechanisms will contribute to tritium retention. The implantation of energetic particles in the plasma-facing components (PFCs), surface adsorption and co-deposition of tritium with material eroded from the PFCs in deposition-dominated areas all contribute to the overall inventory [3]. For the foreseen long pulse operations in ITER, it is expected that retention from the first two mechanisms will rapidly reach saturation and that co-deposition will be the main source of tritium accumulation in the vessel [4].

The innovative PFC materials selection in ITER utilizes beryllium in the main chamber, tungsten in the divertor and carbon for the divertor targets. Thus, co-deposits of isotopic hydrogen and varying mixtures of Be, $\mathrm{W}$, and $\mathrm{C}$ are to be expected in ITER. The composition of co-deposits will depend on the incident species mix and location within the vessel. Be rich co-deposits are seemingly most likely in ITER as the scrapeoff plasma will contain a substantial eroded Be fraction (up to 0.1) from first wall erosion.

Co-deposition of carbon with hydrogen is known to lead to significant retention as was observed in the TFTR [5] and JET [6] tokamaks. Tokamak co-deposition studies involving Be and $\mathrm{W}$ are unavailable but work conducted in plasma devices offers some insight into the retention properties of Be and W co-deposited layers. Experiments in Beseeded PISCES-B plasmas [7, 8], for example, show the formation of Be-rich co-deposits in line-of-sight locations from exposed graphite targets. The co-deposits contained little, if any, carbon and hydrogen retention was found to depend most critically on the substrate temperature of the location where deposits were formed. For ITER, a clear understanding of the retention properties of Be co-deposited layers, and of the expected conditions where such co-deposits may form, is thus of the highest importance for reliable predictions of the in-vessel retention and also for the development of tritium removal techniques [9].

The phenomenon of hydrogen co-deposition with carbon has been extensively investigated and is somewhat better understood. The hydrogen content of $\mathrm{C}$ codeposited layers depends on the energy of the incident particles and on the substrate temperature during the deposition. For incident particle energies above $100 \mathrm{eV}$ hard films with $\mathrm{H} / \mathrm{C} \sim 0.4$ are formed at room temperature, while layers deposited using a low temperature plasma (with low energy of the incident particles) have a higher hydrogen content $(\mathrm{H} / \mathrm{C} \sim 0.8-1)$ [10, 11]. Deuterium retention in tungsten layers have been scarcely 
studied. It was reported in [12], that although not measurable, the deuterium content in co-deposited tungsten layers should be below a few percent. In contrast, a more recent study [13], found $\mathrm{H} / \mathrm{W}$ values of about 0.15 for a tungsten layer formed in a hydrogen plasma.

For the case of beryllium layers, several studies have been carried out focusing mainly on the effect of the substrate temperature on retention. Figure 1 shows the $\mathrm{D} / \mathrm{Be}$ values of the 4 different studies $[12,14,15,16]$ as a function of the substrate temperature during deposition. It is evident that a large scatter exists in the different reported retention values despite similar experimental conditions. One explanation of the differences between Causey [14, 15] and Mayer [12] data was proposed in [3]. The very low deposition rate in [12] lead to the formation of stoichiometric BeO layers, whereas in [15] the layers contain less than $15 \%$ of oxygen. Thus it was proposed that beryllium and hydrogen do not co-deposit to form hydrogen-rich layers but hydrogen and beryllium oxide do. However, the data described in [16] showed D/Be ratio significantly lower than previously reported values despite an oxygen level of about $30 \%$ in layers deposited at $573 \mathrm{~K}$. Such discrepancies, mainly due to a lack of understanding of the co-deposition process, will nevertheless lead to difficulties in the accurate prediction of the retention in ITER and highlight the need for a systematic studies of the influence of the deposition conditions on retention.

This paper describes a first attempt to conduct such a study. Experimental parameters such as the beryllium deposition rate, the incident particle energy, and the substrate temperature are explored and are all shown to affect the level of hydrogen isotope retention in co-deposited beryllium layers. From these results, an empirical relation is established and is shown to be able to predict the $\mathrm{D} / \mathrm{Be}$ values reported in the literature from various plasma devices.

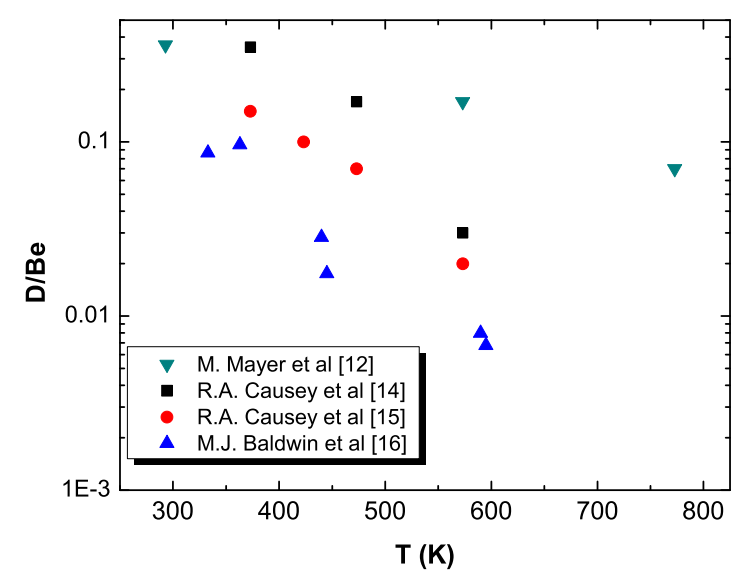

Figure 1. D/Be ratios measured on co-deposited beryllium layers available in the literature. 


\section{Experimental}

Co-deposited beryllium deuterium layers were produced in the PISCES-B linear plasma device. The plasma is generated by an arc discharge initiated with a heated $\mathrm{LaB}_{6}$ cathode. The anode and axial magnetic field define a cylindrical plasma with a radius of about $50 \mathrm{~mm}$ and axial length greater than $1 \mathrm{~m}$. The machine is installed in a sealed enclosure to allow safe operations with beryllium [17].

The experimental setup used has been described in detail in [16] and is summarized only briefly here. A beryllium, or tungsten, target is exposed to a PISCES-B highflux deuterium plasma $\left(2-4 \times 10^{22}\right.$ ions $\left.\cdot \mathrm{m}^{-2} \mathrm{~s}^{-1}\right)$. During plasma exposure, the target temperature is monitored from the rear by a thermocouple. The target temperature is varied in the range 300-900 $\mathrm{K}$ depending on the plasma conditions, but is not found to influence the formation of co-deposits or its retention properties, as co-deposits are collected in a region far from the target. A negative bias, with respect to the plasma potential, is applied to the target to control the energy of ions impacting on the target. A beryllium impurity fraction in the plasma is generated by an evaporative atomic beam source (Veeco/Applied EPI Molecular Beam Epitaxy effusion cell). The plasma parameters, as determined by a reciprocating double Langmuir probe $\left(\mathrm{n}_{e} \sim 2-3 \times 10^{18}\right.$ $\left.\mathrm{m}^{-3}, \mathrm{~T}_{e} \sim 6-10 \mathrm{eV}\right)$, are sufficient to ionize the evaporated beryllium atoms once they enter the plasma. The beryllium ions are thus entrained by the magnetized plasma flow incident on the target. Adjustment of the effusion cell temperature allows independent control of the beryllium fraction in the plasma. In this way, co-deposits of Be and D can be collected by a substrate surface with line of sight to the target. Sputter eroded target $\mathrm{Be}$, and deuterium reflected from the target are the most significant contributions to these co-deposits.

Co-deposits are collected on polished tungsten samples that are used as substrates for beryllium deposition. The substrates are installed on a movable deposition probe assembly and are shielded from cross-field plasma transport. The deposition probe can be independently heated (up to $573 \mathrm{~K}$ ) and is fully retractable into a vacuum interlock chamber to allow fast sample replacement.

The total $\mathrm{D}$ and Be amounts were determined through nuclear reaction analysis (NRA) utilizing the $\mathrm{D}\left({ }^{3} \mathrm{He}, \mathrm{p}\right)^{4} \mathrm{He}$ and the $\mathrm{Be}\left({ }^{3} \mathrm{He}, \mathrm{p}\right){ }^{11} \mathrm{~B}$ reactions. The samples were probed at two primary ${ }^{3} \mathrm{He}$ energies of 0.8 and $1.6 \mathrm{MeV}$. Reaction protons were measured with a semiconductor surface barrier detector of solid angle 0.15 sr. A $12 \mu$ m mylar foil, used to stop scattered ${ }^{3} \mathrm{He}$ and heavy nuclear reaction products, allows only the protons from the reaction into the detector. A ${ }^{3} \mathrm{He}$ fluence of $10 \mu \mathrm{C}$ was a good compromise between counting statistics and particle induced release of the $\mathrm{D}$ during the measurement. The $\mathrm{Be}\left({ }^{3} \mathrm{He}, \mathrm{p}\right){ }^{11} \mathrm{~B}$ reaction is only observable at a primary energy of 1.6 MeV. The spectra were analyzed by converting the peak integrals to D and Be areal densities using the NRA cross section from [18] for D and [19] for Be, respectively.

In addition to NRA, co-deposited layer retention was also measured using thermal desorption mass spectrometry (TDS). Each of the samples was separately desorbed in 
a TDS chamber of typical pressure better than $10^{-6} \mathrm{~Pa}$. An infrared heat source is programmed to linearly increase the sample temperature at a rate of $18 \mathrm{~K} \cdot \mathrm{min}^{-1}$ up to $1100 \mathrm{~K}$ and hold at that temperature for a further period of 10 minutes before cooling at the same rate as the heat up phase. Prior to deposition, the samples are ultrasonically cleaned using acetone then alcohol, and then outgassed using the same procedure used for TDS measurements. This ensures that there is no naturally occuring residual mass 4 inventory in samples prior to forming the co-deposits.

During co-deposition, the energy of the incident particles on a substrate tungsten sample was controlled by changing the negative bias applied on the target. This is because the average energy of the neutrals reflected from the target is related to [20]:

$$
E_{\text {mean }}\left(E_{0}\right)=E_{0} \frac{R_{E}\left(E_{0}\right)}{R_{N}\left(E_{0}\right)}
$$

where $E_{0}$ is the energy of the ions impinging on the target, $R_{N}$ is the fraction of backscattered particles and $R_{E}$ the energy reflection coefficient. Through the use of this expression, and the variation of target bias on beryllium of $-50 \mathrm{~V}$ to $-150 \mathrm{~V}$ and a $-100 \mathrm{~V}$ target bias on tungsten, it was possible to vary the average energy of target reflected (and thus co-deposited) deuterium particles in the range $15 \mathrm{eV}$ to $62 \mathrm{eV}$.

\section{Results}

Figure 2 shows a summary of the data obtained during this study. The D/Be ratio is plotted against the deposition rate of beryllium, which is measured after the experiment by dividing the amount of deposited beryllium, determined by NRA, by the total exposure time. The data are plotted with different symbols according to the incoming particle energy and deposition substrate temperature.

Figure 2 shows that a large range of $\mathrm{D} /$ Be values can be obtained depending on the deposition conditions. D/Be ratios as high as 0.7 are even possible and represent values much higher than previously reported in the literature. Increased surface temperature during deposition, or increased deposition rate of beryllium is observed to lead to a decreased $\mathrm{D} / \mathrm{Be}$ ratio. On the other hand, retention increases with increasing energy of the incident particles. Thus the retention level in co-deposited layers appears to be strongly dependent on the layer formation conditions.

It is found that the data set of figure 2 can be better represented through the use of an empirical scaling, and that this scaling provides some insight into the physics of retention in beryllium co-deposited layers. In order to derive an empirical law describing the evolution of the $\mathrm{D} / \mathrm{Be}$ ratio as a function of the deposition conditions, it is necessary to examine each series of data for which only one parameter is varied, everything else being constant. A regression analysis of these data sets is then made to determine the dependence of the $\mathrm{D} / \mathrm{Be}$ ratio on this given parameter. Since it is assumed that

the deuterium retention is a function of the beryllium deposition rate, the surface temperature, and the energy of the incoming particles, and that the different variables are independent, we write: 


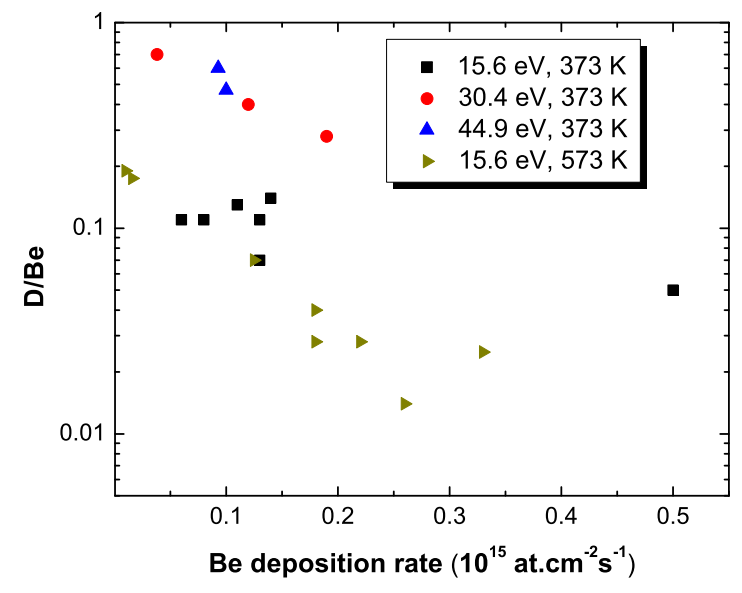

Figure 2. The D/Be ratios measured in co-deposited beryllium layers of this study. The quoted temperature values of $373 \mathrm{~K}$ and $573 \mathrm{~K}$ represent mean values. Actual sample to sample temperature variations can be as high as $\pm 25 \mathrm{~K}$.

$$
\frac{D}{B e}=C \times r_{d}^{\alpha} \times E_{n}^{\beta} \times \exp \left(\frac{\gamma}{T}\right)
$$

where, $\mathrm{C}$ is a constant, $r_{d}$ is the beryllium deposition rate, $E_{n}$ is the average energy of the incoming particles and $T$ the surface temperature.

Figure 3 shows the evolution of the $\mathrm{D} / \mathrm{Be}$ ratio as a function of the deposition rate of beryllium for three different experimental conditions. From the fits to these data it is found that $\frac{D}{B e} \sim r_{d}^{-0.55 \pm 0.15}$ (i.e. $\alpha=-0.55$ in equation (2)). Good agreement is found between the data obtained for $E_{n}=15.6 \mathrm{eV}$ and $T=573 \mathrm{~K}$ and $30.4 \mathrm{eV}$ and $373 \mathrm{~K}$ respectively. The slope of the curve obtained at low temperature and energy appears to be slightly lower than that determined from the two other series of data, but the uncertainty in this slope is still consistent with the slope values of the other two data sets.

To determine the dependence on the energy of the incident particles, experiments were carried out with different bias voltages on the target. In order to account for the influence of the deposition rate, figure 4 shows values of the $\mathrm{D} / \mathrm{Be}$ ratio plotted against the product $E_{n} \times R_{d}^{-0.55}$ ((i.e. $\beta=1.15$ in equation (2)). A fit to this data yields $\frac{D}{B e} \sim E_{n}^{1.15 \pm 0.15}$. It therefore appears that retention increases almost linearly with the energy of the incident deuterium particles, at least in the energy range investigated in this study.

The dependence of the deuterium retention on the substrate temperature is assumed to be of an Arrhenius type, since the desorption of gases from a metal surface is a thermally activated process [21]. To take into account the variation of $\mathrm{D} / \mathrm{Be}$ induced by the different deposition rates and energies, the $\mathrm{D} / \mathrm{Be}$ ratio is divided by the product $E_{n}^{1.15} \times R_{d}^{-0.55}$, and then plotted as a function of the reciprocal temperature, the obtained 


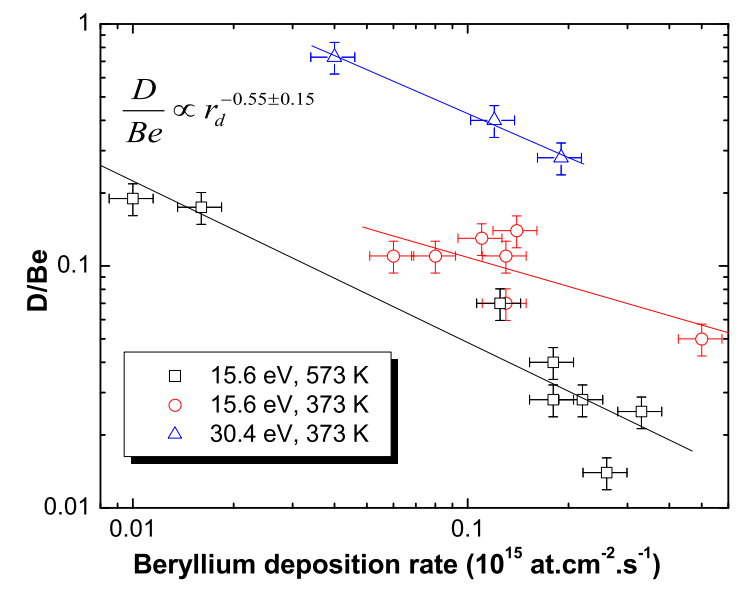

Figure 3. D/Be ratio dependence on the beryllium deposition rate. The series of data used have been obtained for different surface temperatures and energy of the incident particles.

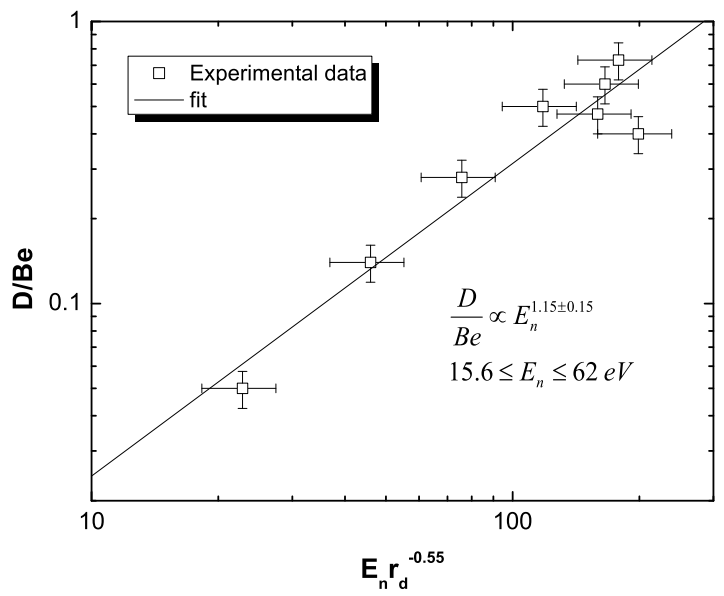

Figure 4. D/Be ratio dependence on the energy of the incident deuterium particles. All the layers were prepared at about $100^{\circ} \mathrm{C}$. The $\mathrm{D} / \mathrm{Be}$ values are plotted as a function of the product $E_{n} \times R_{d}^{-0.55}$ to account for the variations of the deposition rate.

graph is shown in figure 5. It is found that $\frac{D}{B e} \sim \exp \left(\frac{1394 \pm 180}{T}\right)$ (i.e. $\gamma=1394$ in equation $(2))$.

Having determined the dependence of $\mathrm{D} / \mathrm{Be}$ on the three studied parameters, the validity of equation (2) is now established. In order to determine the proportionality constant $\mathrm{C}$ introduced in equation (2) and to determine more accurately the parameters $\alpha, \beta$, and $\gamma$, a regression analysis is performed on all of the data. This allows to take into account the dependence on each individual parameters more precisely than what was 


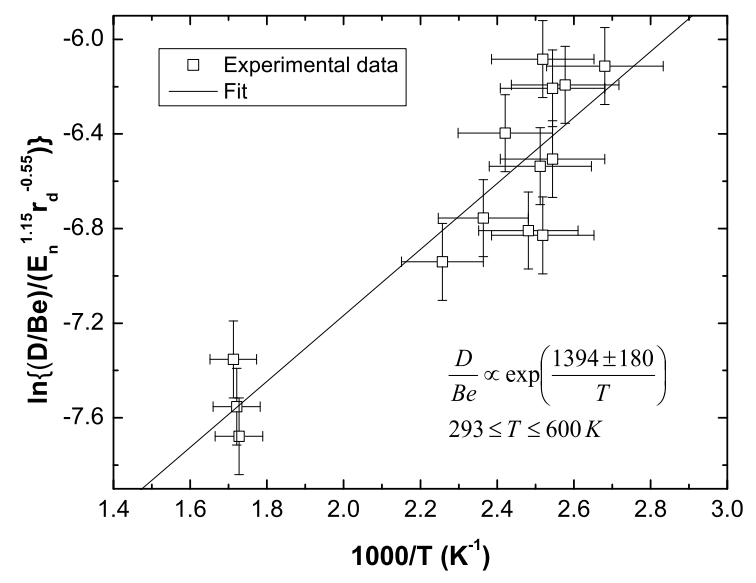

Figure 5. D/Be ratio dependence on the substrate temperature during deposition. To account for the different incident particles energies and deposition rates, the $\mathrm{D} / \mathrm{Be}$ is divided by $E_{n}^{1.15} \times R_{d}^{-0.55}$.

done in figures 3-5. This is shown in figure 6 , where the experimentally measured D/Be ratio is plotted against the scaling term $E_{n}^{1.34} \times R_{d}^{-0.59} \times \exp \left(\frac{1306}{T}\right)$, determined from the regression analysis. Evidently, a linear fit to the scaled data yields a good agreement between the experimental values and the scaling factor. Note that the exponent of each parameter is slightly different to that determined from the dependence on each individual parameter. The full scaling expression derived from fitting all of the data is thus:

$$
\frac{D}{B e}=2.94 \cdot 10^{-5} \times r_{d}^{-0.59 \pm 0.1} \times E_{n}^{1.34 \pm 0.15} \times \mathrm{e}^{\frac{1306 \pm 190}{T}}
$$

where $r_{d}$ is in units of $10^{15} \mathrm{at} \cdot \mathrm{cm}^{-2} \cdot \mathrm{s}^{-1}, E_{n}$ in eV and $T$ in $\mathrm{K}$, and with $293 \mathrm{~K} \leq T \leq$ $600 \mathrm{~K}$ and $15.6 \mathrm{eV} \leq E_{n} \leq 62 \mathrm{eV}$.

It is pointed out, however, that this equation is strictly an empirical expression that displays seemingly good agreement with measured D/Be values of co-deposits produced in the PISCES-B device. In the following section the scaling of equation (3) is explored further in relation to the mechanisms that influence retention in co-deposits, and its applicability to other experimental systems.

\section{Discussion}

\subsection{Mechanisms that affect retention in co-deposits}

The fact that the $\mathrm{D} / \mathrm{Be}$ ratio decreases with increasing beryllium deposition rate is not too surprising. It is a common trend in vacuum deposition techniques to find that the impurity content in a deposited layer is reduced with increased deposition rate. This has been observed both for inert gas entrapment in growing films and for reactive 


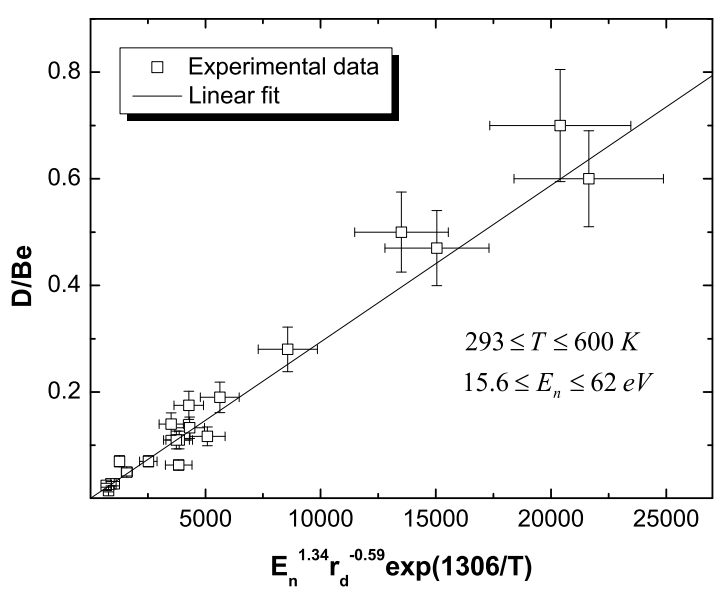

Figure 6. Comparison between experimentally determined D/Be ratio and the values of the scaling expression. A linear fit to the data yields the determination of the constant introduced in equation (2).

species (such as oxygen in metal films), and it is found that impurity levels are inversely proportional to the deposition rate $[27,28]$. In the present experiments, the $\mathrm{D} / \mathrm{Be}$ ratio varies as $r_{d}^{-0.59}$. This value is quite different from what is reported in [27, 28], but it is pointed out that the current experiment is substantially more complex, because of the presence of a plasma, and that this may not allow direct comparison to vacuum deposition experiments.

Increased deuterium retention with ion energy has been observed for bulk beryllium both during ion beam experiments and plasma exposures [22]. A similar trend is observed in the experiments described here, and is represented by an almost linear variation of the deuterium retention as a function of the energy of the incident particles. It should be emphasized that no saturation in deuterium retention has been observed for the layers investigated here, but $\mathrm{D} /$ Be ratios as high as 0.7 have been produced. This is much different to implanted beryllium where the deuterium retention does saturate and the corresponding $\mathrm{D} / \mathrm{Be}$ ratio in the implant zone is in the range 0.3-0.4 [23, 24]. It is intriguing as to the mechanism behind such high retention. No blisters have been observed by SEM on the co-deposited beryllium layers, but high levels of porosity are observed [25].

The Arrhenius temperature dependence in equation (3) accounts for the fact the desorption rate of a gas from a metal is a thermally activated process characterized by an activation energy for desorption, $E_{\text {des }}$. The value of $\gamma=1306 \pm 190 K$ yields an activation energy of $11.5 \pm 0.15 \mathrm{~kJ} / \mathrm{mol}$ and agrees relatively well with the desorption energy of $14.4 \mathrm{~kJ} / \mathrm{mol}$ for press-sintered beryllium exposed to deuterium plasma [26], and that for data obtained in [16] for co-deposited beryllium layers.

In [12] the term co-implantation was proposed to explain the deuterium retention in 
BeO layers. However, the observed dependence of $\mathrm{D} / \mathrm{Be}$ on both the deposition rate and incident particle energy implies that retention in Be co-deposits cannot be described as a pure co-deposition, or implantation mechanism, but proceeds rather by a combination of both mechanisms. Indeed, in equation (3), the decrease of the retention with increasing deposition rate suggests a co-deposition mechanism. Once trapped though, the retained D inventory can reach alarming levels, but at this point the nature of the trapping remains for further investigations. On this note though, it is worth mentioning that beryllium can in fact form a hydride $\left(\mathrm{BeH}_{2}\right)$ [29] whose direct preparation from the elements is not possible and usually requires a complex process using organo-beryllium compounds.

\subsection{Applicability of the scaling expression to literature data}

As shown in the present study, the deuterium retention in beryllium layers strongly depends on the deposition conditions. Equation (3) provides a convenient way to predict the retention in PISCES-B co-deposits when these conditions are taken into account. However, the usefulness of this expression may not be limited to PISCES-B and it is pointed out that other than the constant, $\mathrm{C}$, none of the scaling terms are generic to the PISCES-B device. To explore this further, the apparently wide-ranging D/Be values of Mayer [12], Causey [14, 15] and Baldwin [16], shown in figure 1, were re-examined with the use of the scaling expression. Only the data for which all the needed information (temperature, deposition rate, energy) could be found in the respective articles were used. One exception is that data from [12] were excluded because of the extremely low reported deposition rate (less than 0.01 monolayer/s), probably caused by the re-erosion of the deposited layer by the energetic incident particles. The three following studies were therefore used.

In [15], co-deposited samples were prepared by collecting beryllium atoms sputtered from a beryllium target exposed to the TPE linear plasma facility. The energy of the incident ions on the target was $-100 \mathrm{eV}$. Under those conditions, the average energy of the deuterium particles impinging on the catcher plate was about $30 \mathrm{eV}$. Experiments were made with different substrate temperatures $373 \mathrm{~K}, 473 \mathrm{~K}$, and $573 \mathrm{~K}$.

In previous PISCES-B experiments [16], a graphite target, biased at $-50 \mathrm{~V}$, was exposed to a beryllium seeded deuterium plasma. The experimental conditions are very close to those described in the present paper. Beryllium layers were formed under identical conditions but with different substrate temperatures in the range 300-573 K. The average energy of the incident particles on the witness plate was about $15 \mathrm{eV}$. It should be mentioned that experiments carried out in PISCES-B and TPE are quite similar given the similarity of both machines.

Finally, in [30] beryllium oxide (BeO) layers were produced by sputtering a beryllium target with $10 \mathrm{keV}$ Ne ions in deuterium gas at a pressure of $1 \mathrm{~Pa}$. Sputtered atoms are deposited on a tungsten substrate located at a distance of $2 \mathrm{~cm}$ from the sputtered spot on the target. The substrate is negatively biased to provide the 
simultaneous bombardment of the growing film surface with deuterium ions generated by $\mathrm{Ne}^{+}-\mathrm{D}_{2}$ collisions. Two different bias voltages were used: $-50 \mathrm{~V}$ and $-400 \mathrm{~V}$. There is however an uncertainty in the composition of the ion flux (a mixture of $\mathrm{D}^{+}, \mathrm{D}_{2}^{+}$and $\mathrm{D}_{3}^{+}$) striking the surface, and thus an uncertainty on the average energy per deuterium atom striking the surface of the growing film.

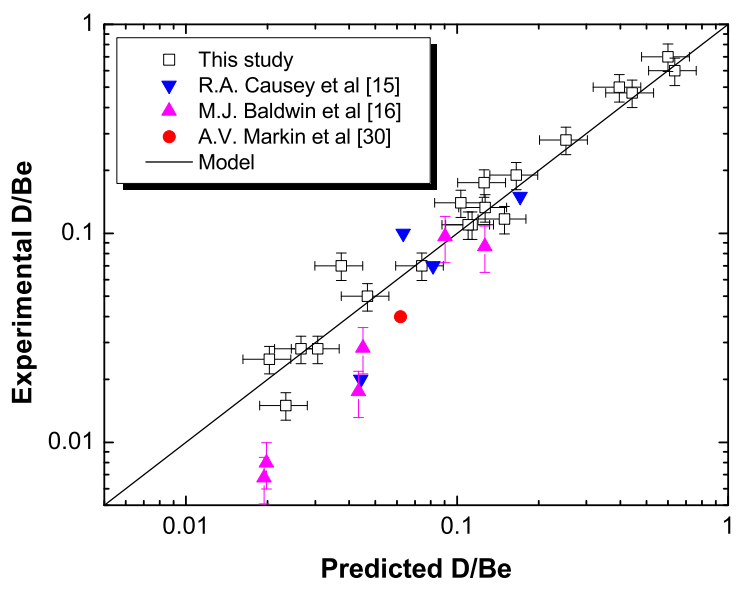

Figure 7. Comparison between experimentally determined D/Be values from different studies and values predicted by equation (3) using the deposition conditions described in the respective references.

A comparison of the experimentally measured $\mathrm{D} / \mathrm{Be}$ ratio found in each of these references with the $\mathrm{D} / \mathrm{Be}$ ratio predicted by equation (3), is shown in figure 7 . For the data extracted from [30], only the data obtained with a bias of $-50 \mathrm{~V}$ are shown. The $400 \mathrm{eV}$ data of [30] have been excluded since the scaling expression determined here is not experimentally determined over a particle energy of $62 \mathrm{eV}$. As shown, a relatively good agreement is observed for the different datasets. Moreover, the value of the constant $\mathrm{C}$ does not change when adding the selected literature data, and therefore appears not to be generic to the PISCES-B data presented here.

It is interesting to note that contrary to what was postulated for example in [22], the oxygen content in the layer does not seem to explain the retention values observed. Indeed data from [30] relates to beryllium oxide layers while the layers created in this study have a low oxygen content (less than 10\%). Using this scaled approach, a reliable comparison of the data from different experiments seems to be possible if the different layer formation conditions are known. Furthermore, the discrepancies described in section 1 between the different published data can be resolved using such an approach as shown by figure 7, and appear to be due to the differing experimental conditions. Indeed, the data from [15] and [16] were obtained under almost similar conditions, yet the higher $\mathrm{D} / \mathrm{Be}$ values obtained in [15] could be due to the higher energy of the deuterium neutrals bombarding the layer during deposition. In a similar way, we can 
possibly say that the high $\mathrm{D} /$ Be values obtained by Mayer [12] are a result of very low deposition rate and the high energy of the incident deuterium particles. It should be noted, that a saturation may be expected in the effect of the particle energy on the retention, which would explain why the high energy values from [30] cannot be included in this study.

In any case, the scaling expression developed here provides a method to predict the retention in Be co-deposits formed in PISCES-B, and also offers reasonable agreement with literature data. Given these ties, it is not unreasonable to expect that the scaling may also be applicable to fusion reactor Be co-deposits. In this case, the scaling expression of equation (3) might be very important in providing predictions of the tritium inventory in co-deposited beryllium layers in ITER.

Hitherto, modelling of the tritium retention was made by assuming a fixed $\mathrm{T} / \mathrm{Be}$ ratio in the deposited beryllium layers [31]. However, as shown in [31], along the divertor targets, the energy of the impinging deuterium particles, as well as the beryllium deposition rate, will vary which, according to the present results implies different levels of retention in the layers.

\section{Conclusions}

The deuterium retention in co-deposited beryllium layers was found to strongly depend on the layer formation conditions: beryllium deposition rate, substrate temperature and energy of incident deuterium particles. The mechanism by which deuterium co-deposits with beryllium appears to be a combination of co-deposition and implantation. Indeed, the retention decreases with increasing deposition rate, which indicates a co-deposition process, and increases with the energy of the impinging deuterium particles, which is a trend usually observed during implantation. An empirical equation has been proposed to account for the influence of those three parameters on the D/Be ratio. This equation is able to reproduce the results obtained in other plasma devices, thus providing an explanation to the discrepancies in the $\mathrm{D} / \mathrm{Be}$ values reported in various studies, and most likely caused by the different layer formation conditions to which the retention is very sensitive. This study has potentially important consequences for the prediction of tritium retention in ITER.

\section{Acknowledgments}

The authors gratefully acknowledge the help and support from the PISCES-B technical staff, with particular mention to T. Lynch for the samples preparation. This work was supported by the US Department of Energy under the contract DE-FG02-07ER54912, and by the US-EU bilateral collaboration on mixed materials. 


\section{References}

[1] Technical basis for the ITER final design, 2002 ITER EDA Documentation series No 24 (radiological source terms), chapter 5.3 (Vienna:IAEA)

[2] Pitts R.A. et al 2005 Plasma Phys. Contr. Fusion 47 B303

[3] Causey R.A. 2002 J. Nucl. Mater. 30091

[4] Haasz A.A. 2004 Nuclear Fusion Research: Understanding Plasma-Surface Interactions p 225 (Springer series in Chemical Physics)

[5] Skinner C.H. et al 2001 J. Nucl. Mater. 290-293 486

[6] Coad J.P. et al 2006 Nucl. Fusion 46350

[7] Baldwin M.J. and Doerner R.P. 2006 Nucl. Fusion 46444

[8] Schmid K. et al 2004 Nucl. Fusion 44815

[9] Counsell G. et al 2006 Plasma Phys. Control. Fusion 48 B189

[10] Reinke P. et al 1992 J. Vac. Sci. Technol. A10 434

[11] Jacob W. 1998 Thin Solid Films 3261

[12] Mayer M. et al 1996 J. Nucl. Mater. 23067

[13] Katayama K. et al 2007 Fusion Eng. Des. 821645

[14] Causey R.A. et al 1996 Proceedings of International Workshop on Present Status and Prospect of Tritium Material Interaction Studies Toyama, Japan, 18-19 July 1996.

[15] Causey R.A. and Walsh D.S. 1998 J. Nucl. Mater. 25484

[16] Baldwin M.J. et al 2005 J. Nucl. Mater. 337-339 590

[17] Doerner R.P. and Mays C. 1997 Fus. Eng. Des. 37325

[18] Alimov V.Kh. et al 2005 Nucl. Instr. Meth. B234 169

[19] Wolicki E.A. et al. 1959 Phys.Rev. 1161585

[20] Eckstein W. 2002 Calculated sputtering, reflection and range values IPP-Report IPP 9/132 (MaxPlanck-Institut fr Plasmaphysik, Garching

[21] King D.A. 1975 Surface Sci. 47384

[22] Anderl R.A. et al 1999 J. Nucl. Mater. 2731

[23] Wampler W.R. 1984 J. Nucl. Mater. 122-123 1598

[24] Haasz A.A. and Davies J.W. 1997 J. Nucl. Mater. 241-243 1076

[25] De Temmerman G. et al 2007 J. Appl. Phys. 102083302

[26] Doerner R.P. et al 2000 Fus. Eng. Des. 49-50 183

[27] Paturaud C. et al 1999 Thin Solid Films 34746

[28] Sobe G. et al 1992 Contrib. Plasma Phys. 32623

[29] Baker R.W. et al 1978 J. Organometallic. Chem. 1593589

[30] Markin A.V. et al 2000 J. Nucl. Mater. 293-287 1094

[31] Kirschner A. et al 2007 J. Nucl. Mater. 363-365 91 